\title{
Chronic Stress Induces a Selective Decrease in AMPA Receptor-Mediated Synaptic Excitation at Hippocampal Temporoammonic-CA1 Synapses
}

\author{
Angy J. Kallarackal, ${ }^{1,3 *}$ Mark D. Kvarta, ${ }^{1,3,4 *}$ Erin Cammarata, ${ }^{5}$ Leelah Jaberi, ${ }^{1}$ Xiang Cai, ${ }^{1}$ Aileen M. Bailey, ${ }^{5}$ \\ and Scott M. Thompson ${ }^{1,2,3}$ \\ ${ }^{1}$ Department of Physiology, ${ }^{2}$ Department of Psychiatry, ${ }^{3}$ Programs in Neuroscience and Membrane Biology, ${ }^{4}$ Medical Scientist Training Program, \\ University of Maryland School of Medicine, Baltimore, Maryland 21201, and 5 Department of Psychology, Saint Mary's College of Maryland, St. Mary's City, \\ Maryland 20686
}

Chronic stress promotes depression, but how it disrupts cognition and mood remains unknown. Chronic stress causes atrophy of pyramidal cell dendrites in the hippocampus and cortex in human and animal models, and a depressive-like behavioral state. We now test the hypothesis that excitatory temporoammonic (TA) synapses in the distal dendrites of CA1 pyramidal cells in rats are altered by chronic unpredictable stress (CUS) and restored by chronic antidepressant treatment, in conjunction with the behavioral consequences of CUS. We observed a decrease in AMPAR-mediated excitation at TA-CA1 synapses, but not Schaffer collateral-CA1 synapses, after CUS, with a corresponding layer-specific decrease in GluA1 expression. Both changes were reversed by chronic fluoxetine. CUS also disrupted long-term memory consolidation in the Morris water maze, a function of TA-CA1 synapses. The decreases in TA-CA1 AMPAR-mediated excitation and performance in the consolidation test were correlated positively with decreases in sucrose preference, a measure of anhedonia. We conclude that chronic stress selectively decreases AMPAR number and function at specific synapses and suggest that this underlies various depressive endophenotypes. Our findings provide evidence that glutamatergic dysfunction is an underlying cause of depression and that current first-line antidepressant drugs act by restoring excitatory synaptic strength. Our findings suggest novel therapeutic targets for this debilitating disease.

\section{Introduction}

Major depression affects $\sim 7 \%$ of the world population. Despite its prevalence and socioeconomic burden, there is no known direct cause or cure. Depression results from an interaction between genetic predisposition and environmental factors, such as chronic stress.

A depressive-like behavioral state is induced in rodents by chronic unpredictable stress (CUS; Willner et al., 1987). This model demonstrates construct and face validity, because stress correlates with human depression and CUS animals exhibit signs similar to those of depressed patients, including anhedonia and decreased social interaction (Willner et al., 1987; Berton and Nestler, 2006). CUS also exhibits predictive validity, because these

Received June 18, 2013; revised Aug. 5, 2013; accepted Aug. 20, 2013.

Author contributions: A.J.K., M.D.K., E.C., X.C., A.M.B., and S.M.T. designed research; A.J.K., M.D.K., E.C., L.J., X.C., and A.M.B. performed research; A.J.K., M.D.K., E.C., L.J., X.C., A.M.B., and S.M.T. analyzed data; A.J.K., M.D.K., X.C., A.M.B., and S.M.T. wrote the paper.

This work was supported by the National Institutes of Health (X.C., S.M.T.). We thank Kaitlin Gaylor and Pamela Gorgei for technical assistance.

*A.J.K. and M.D.K. contributed equally to this work.

Correspondence should be addressed to Scott M. Thompson at the above address. E-mail: sthom003@umaryland.edu.

A. J. Kallarackal's present address: Department of Biology, University of Utah, Salt Lake City, UT 84112. X. Cai's present address: Department of Physiology, Southern Illinois University, Carbondale, IL 62901.

DOI:10.1523/JNEUROSCI.2588-13.2013

Copyright $\odot 2013$ the authors $\quad 0270-6474 / 13 / 3315669-06 \$ 15.00 / 0$ signs are reversed by antidepressants (ADs) when administered chronically (Bondi et al., 2008).

McEwen and others have established that chronic stress results in physical changes in rodent brains. In the hippocampus, chronic stress causes loss of distal apical dendritic branches in CA3 pyramidal cells and decreases in the size and number of dendritic spines (Watanabe et al., 1992; Magariños and McEwen, 1995; McEwen, 1999; Sousa et al., 2000; Vyas et al., 2002), but its effects on CA1 cells are less well characterized. Although stressinduced impairment of excitatory synapses has been described in the medial prefrontal cortex (Yuen et al., 2012) and nucleus accumbens (Lim et al., 2012), the evidence of changes in excitation in hippocampus is ambiguous. In area CA3, chronic stress enhances synaptic currents mediated by NMDA-type glutamate receptors (NMDARs), but has no effect on AMPA-type receptors (AMPARs; Kole et al., 2002). In contrast, chronic stress has no effect on basal synaptic strength at Schaffer collateral (SC) synapses in area CA1, but does impair LTP (Alfarez et al., 2003). Finally, chronic social stress results in decreases in mRNA for GluA1 AMPAR subunits in CA1 (Schmidt et al., 2010). The functional consequences of stress-induced dendritic atrophy are thus unclear.

We have shown that serotonin potentiates glutamatergic transmission at temporoammonic-CA1 (TA-CA1), but not SCCA1, synapses. This potentiation is quantitatively and qualitatively altered after CUS (Cai et al., 2013). We suggested that 
chronic stress induces changes in excitatory synapses and that dysfunction of excitatory synaptic transmission contributes to the genesis of depression. We now test this hypothesis using TA-CA1 synapses as a model.

\section{Materials and Methods}

CUS. Male Sprague Dawley rats (3-4 weeks old at start; Harlan Laboratories) were randomly divided into control and CUS groups. CUS animals were treated with a $7 \mathrm{~d}$ cycle of stressors (Cai et al., 2013), repeated over 6-7 weeks.

$A D$ treatment. Animals were given fluoxetine $(80 \mathrm{mg} / \mathrm{ml})$ via their drinking water during the last 3-4 weeks of CUS. All animals were housed singly and water was changed every $3 \mathrm{~d}$. Control animals received water only.

Sucrose preference test. Rats were presented with two bottles containing tap water or water containing $1 \%$ sucrose. Animals were first trained with both bottles while group-housed. For subsequent tests, including during the baseline period, animals were individually housed. Only animals displaying an initial sucrose preference $>65 \%$ were used for further experiments.

Novelty-suppressed feeding. Novelty-suppressed feeding tests were performed in a brightly lit arena in a dimly lit room (Santarelli et al., 2003). Food pellets were placed on white paper in the center of the box. Rats were food-deprived for $24 \mathrm{~h}$. A maximum time allowance was set at $600 \mathrm{~s}$. Animals were then returned to their home cage and allowed to feed for 5 min. Food pellets were weighed before and after the $5 \mathrm{~min}$ to calculate food consumption.

Acute slice electrophysiology. Standard methods were used to prepare $400-\mu \mathrm{m}$-thick transverse hippocampal slices. Dissection and recording saline contained the following: $124 \mathrm{~mm} \mathrm{NaCl}, 3 \mathrm{~mm} \mathrm{KCl}, 1.25 \mathrm{~mm}$ $\mathrm{NaH}_{2} \mathrm{PO}_{4}, 1.5 \mathrm{~mm} \mathrm{MgCl}, 2.5 \mathrm{~mm} \mathrm{CaCl}_{2}, 26 \mathrm{~mm} \mathrm{NaHCO}_{3}$, and $10 \mathrm{~mm}$ glucose, bubbled with $95 \% \mathrm{O}_{2} / 5 \% \mathrm{CO}_{2}$. Slices were then transferred to a submersion-type recording chamber and perfused at $20-22^{\circ} \mathrm{C}$. Picrotoxin $(100 \mu \mathrm{M})$ and CGP52432 $(2 \mu \mathrm{M})$ were included to block $\mathrm{GABA}_{\mathrm{A}}$ and $\mathrm{GABA}_{\mathrm{B}}$ receptors, and the dentate gyrus and $\mathrm{CA} 3$ region were removed.

Because TA-CA1 synapses are electrotonically remote from CA1 cell somata, we used extracellular recording of local field EPSPs (fEPSPs). Recording pipettes contained saline (3-5 M $\Omega$ ) and were placed in stratum lacunosum-moleculare (SLM) to record TA-CA1 responses and/or stratum radiatum (SR) to record SC-CA1 responses. fEPSPs were amplified $1000 \times$, filtered at $3 \mathrm{kHz}$, and digitized at $10 \mathrm{kHz}$. Concentric bipolar tungsten electrodes were placed $>500 \mu \mathrm{m}$ from the stimulating electrodes in SLM for TA afferents or SR for SC afferents. Stimuli $(100 \mu \mathrm{s})$ were delivered at $0.05 \mathrm{~Hz}$. In most experiments, the stimulus intensity was set at $150 \%$ of threshold intensity, resulting in a fEPSP of $0.1-0.2 \mathrm{mV}$.

For AMPA/NMDA ratios, $\mathrm{Mg}^{2+}$-free saline was used to produce a robust NMDAR-mediated component of the fEPSP. Three consecutive responses were averaged and fEPSP slope was calculated over a 1-3 ms window. For AMPAR-mediated responses, the window was fixed in the initial rising phase of the response, $2-5 \mathrm{~ms}$ after its initiation. For NMDA responses, DNQX $(50 \mu \mathrm{M})$ was applied for $15 \mathrm{~min}$ and the slope was calculated over a $3-5 \mathrm{~ms}$ window in the rising phase of the response, 5-10 $\mathrm{ms}$ after its initiation.

Western blotting. SLM or SR tissue punches (1 mm diameter) were dissected from area CA1 in hippocampal slices and pooled (3-4). Membranes were probed with antibodies against GluA1 $(0.5 \mu \mathrm{g} / \mathrm{ml}$; Millipore Bioscience Research Reagents), GluN1 (1:1000; Millipore), PSD-95 (1: 1000; Millipore), or $\beta$-actin (1:2000; Cell Signaling Technology). Levels of proteins are expressed as the ratio of intensity normalized to $\beta$-actin intensity.

Memory consolidation. Rats were trained as described previously (Remondes and Schuman, 2004; Cai et al., 2013). Animals received 10 blocks of training (four trials/block) over $6 \mathrm{~d}$. The platform remained in a fixed location throughout, relative to spatial cues surrounding the tank. Twenty-four hours following the 10th block of training, animals completed a probe trial and were returned to the animal facility for $28 \mathrm{~d}$ or subjected to CUS. Long-term consolidation was then tested with a probe trial $28 \mathrm{~d}$ after completing the 10th block of training.

Statistics. Data are presented as mean \pm SEM. Experiments were performed and analyzed with the experimenter blinded to the prior treatment.

\section{Results \\ Selective attenuation of AMPAR-mediated responses at TA-CA1 synapses after CUS}

We first compared fEPSPs in SLM in area CA1 in response to stimulation of TA afferents in acute slices from control rats, rats subjected to 6-7 weeks of CUS, and rats treated with fluoxetine during the last 3 weeks of CUS. Sucrose preference test (SPTs) were performed in all animals. For these experiments, only CUS rats with sucrose preferences $<65 \%$ and control and fluoxetinetreated animals with $>65 \%$ sucrose preference were compared.

We observed no difference in the paired-pulse ratio of TACA1 fEPSPs in slices from control and CUS animals (control: $1.51 \pm 0.1, n=19$; CUS: $1.44 \pm 0.1 ; n=14 ; p>0.5, t$ test), suggesting that presynaptic release probability at TA nerve terminals was not affected by CUS, in agreement with previous reports (Kole et al., 2002; Yuen et al., 2012). AMPAR-mediated responses were considerably diminished in slices from CUS rats, compared with controls, using two independent methods to control for the intensity of stimulation and the health of the slices (fiber volley amplitude and NMDAR-mediated responses). We first normalized the AMPAR responses to the amplitude of fiber volley over a range of stimulation intensities. The slope of this relationship was then determined for each slice. The mean slope was significantly $(p<0.05$, Mann-Whitney $U$ test) lower in CUS slices $(n=10$ slices), compared with control slices $(n=8$; Fig. $1 a, b)$. Similarly, when we normalized responses by comparing the slopes of the AMPAR-mediated and NMDAR-mediated components at a stimulation intensity producing fiber volleys of $\sim 0.15 \mathrm{mV}$ before and after application of DNQX, the AMPA/NMDA ratio was significantly ( $p<0.01$, Mann-Whitney $U$ test) lower in CUS slices compared with controls (Fig. 1c). This difference did not result from a difference in NMDAR-mediated responses because the relationship between NMDAR-mediated responses and fiber volley amplitude was not significantly different in any group (Fig. 1b).

Chronic stress-induced changes in behaviors can be reversed by 3 weeks of fluoxetine administration (Muscat et al., 1992; Cai et al., 2013). Consistent with the recovery of normal behavior, AMPA/fiber volley (Fig. 1b) and AMPA/NMDA (Fig. 1c) ratios were not significantly different in slices from control rats and slices from rats subjected to CUS and then administered fluoxetine ( $n=8,8$ slices).

The decrease in basal synaptic strength at TA-CA1 synapses of rats subjected to CUS was not accompanied by a relative increase in LTP. We found no substantial difference in either the magnitude or the time course of LTP at TA-CA1 synapses in slices from CUS rats compared with control rats in response to strong highfrequency stimulation trains (Fig. 1d).

To determine whether the decrease in AMPAR-mediated excitation was due to changes in receptor number or function, we harvested SLM tissue in slices from controls, rats subjected to CUS, and rats subjected to CUS and then treated with fluoxetine. Consistent with the electrophysiology, GluA1 protein was significantly decreased in SLM in CUS tissue compared with control tissue and fluoxetine-treated CUS tissue ( $n=6,6,4$ slices; Fig. $2 a$ ), whereas GluA2 and GluN1 expression were unaffected (Fig. 2b). Consistent with atrophy of dendritic spines, we observed signif- 
a
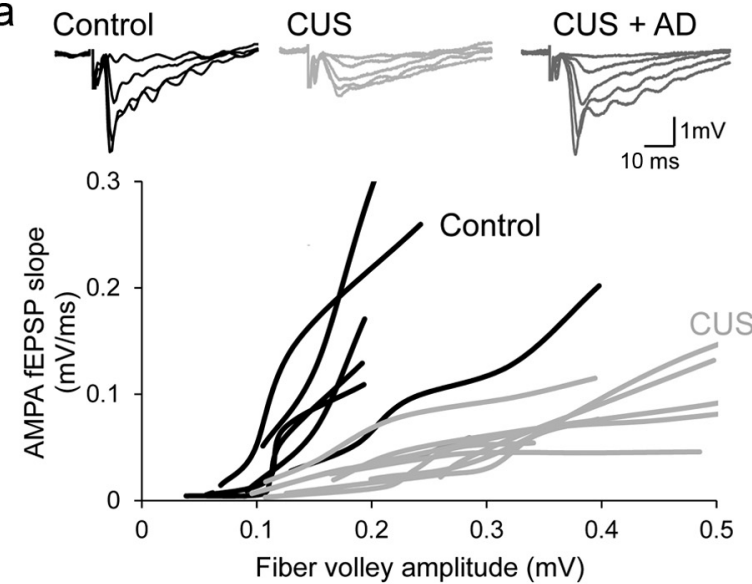

C Control
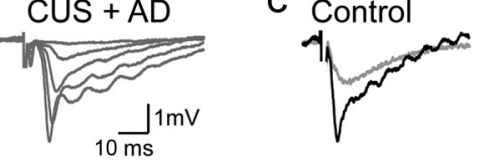
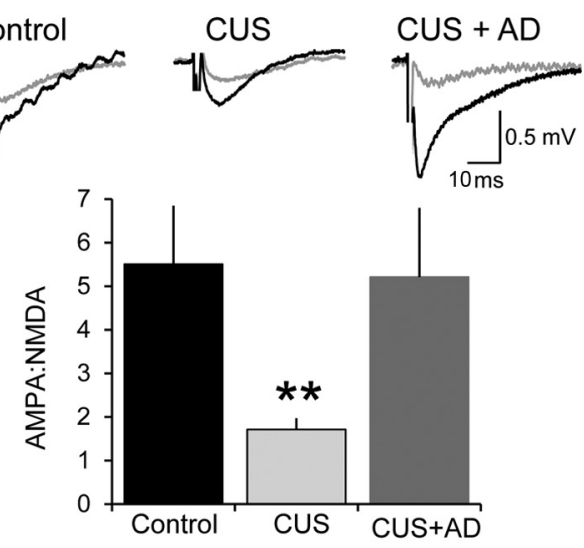

b

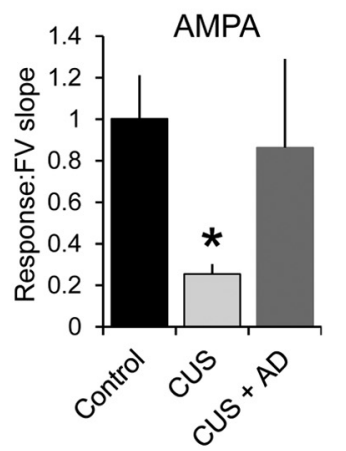

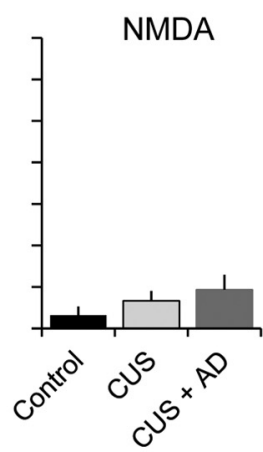

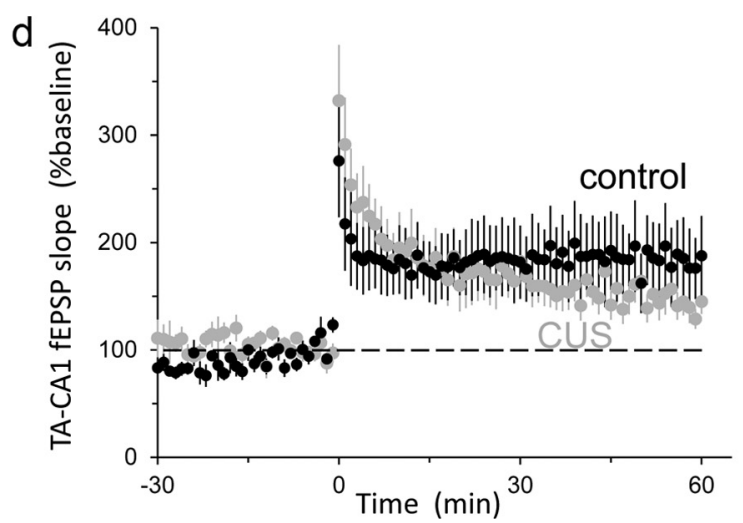

Figure 1. Chronic stress impairs AMPAR-mediated synaptic excitation at TA-CA1 synapses. $\boldsymbol{a}$, fEPSPs recorded in SLM in response to stimuli delivered at a graded series of intensities in Mg ${ }^{2+}{ }_{-}$-free saline. Each line represents a series of responses from one slice from control (black) or CUS (gray) rats. Sets of data from representative slices shown above. $\boldsymbol{b}$, Mean of the slopes of straight line fits to the data in $\boldsymbol{a}$ in control rats $(n=6)$, CUS rats $(n=6)$, and CUS rats administered fluoxetine $(n=8)$ in $\mathrm{Mg}^{2+}$-free saline before (left) and after (right) addition of DNQX. $c$, Mean of the slope of the AMPAR-mediated and NMDAR-mediated components taken from each slice at a stimulation intensity that produced a fiber volley of $\sim 0.15 \mathrm{mV}$. $\boldsymbol{d}$, TA-CA1 fEPSP slope is plotted before and after induction of LTP in slices from control (black, $n=8$ slices) and CUS animals (gray, $n=11$ slices). ${ }^{*} p<0.05,{ }^{* *} p<0.01$.
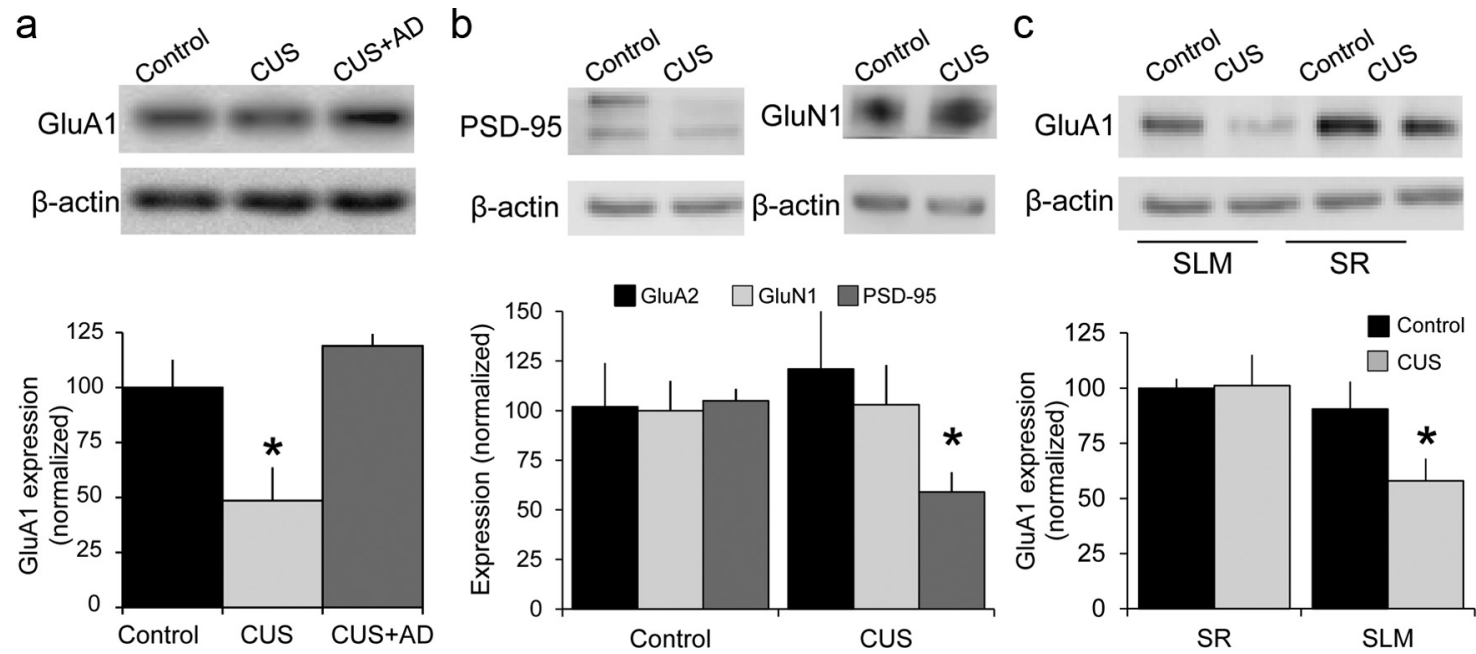

Figure 2. Chronic stress decreases Glu1 expression selectively. $\boldsymbol{a}$, GluA1 expression, normalized to $\beta$-actin levels. Samples taken from SLM in slices from control rats ( $n=6$ ), CUS rats ( $n=6$ ), and CUS rats administered fluoxetine ( $n=4)$. $\boldsymbol{b}$, PSD-95 ( $n=5$ control, 6 CUS), but not GluA2 or GluN1 $(n=8,8,9)$ expression, was decreased in SLM in CUS tissue compared with controls. c, GluA1 expression was decreased selectively in SLM $(n=7,6)$ in CUS tissue compared with controls, but not in $\operatorname{SR}(n=5,5)$. ${ }^{*} p<0.05$, Mann-Whitney $U$ test.

icant decreases in expression of the postsynaptic protein PSD95 in SLM in CUS tissue compared with controls (Fig. $2 b$ ).

The decrease in AMPAR-mediated excitation was specific to TA-CA1 synapses. We compared ratios in the same slices, delivering alternating stimuli, and observed no significant differ- ences between SC-CA1 AMPA/NMDA ratios in control and CUS animals (Fig. $3 a$ ) and no differences in GluA1 expression in SR (Fig. 2c).

There was a strong correlation between synaptic strength and chronic stress-induced changes in behavior. Rats subjected to 

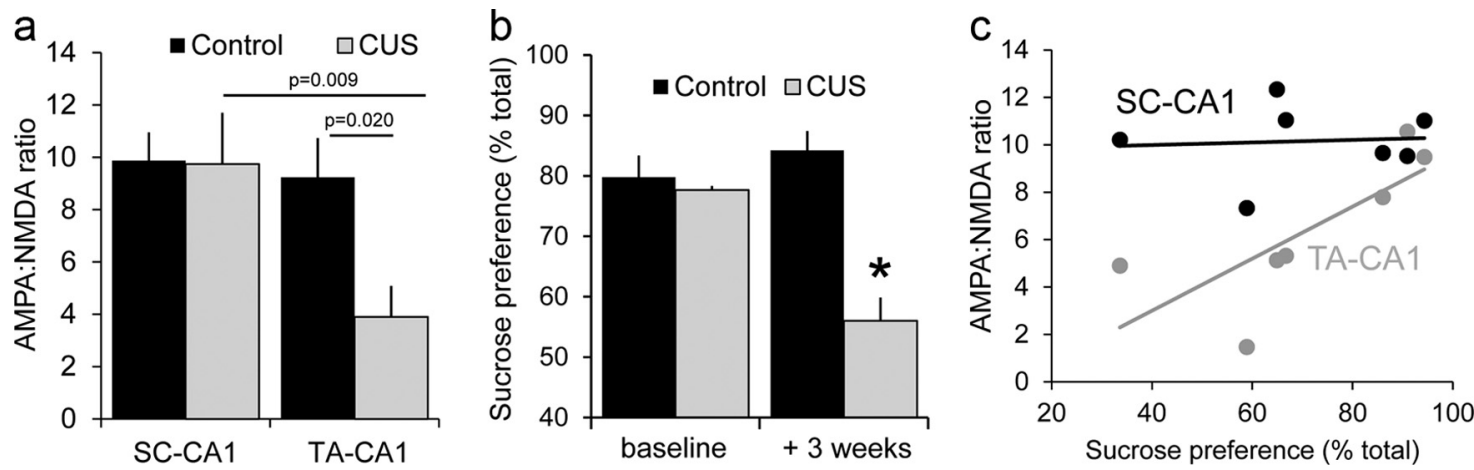

Figure 3. Decreases in AMPAR-mediated excitation are specific to TA-CA1 synapses and correlated with affective state. $\boldsymbol{a}$, Mean AMPA/NMDA ratios for SC-CA1 and TA-CA1 fEPSPs in slices from control and CUS rats (synapse- group interaction, $F_{(1,8)}=7.525, p=0.025$ ). There was no difference between control and CUS at SC-CA1 synapses, whereas the ratio for TA-CA1 responses was significantly different for both CUS SC-CA1 and control TA-CA1 responses. $\boldsymbol{b}$, Sucrose preference before and after 3 weeks in control and CUS rats. The difference was significant for CUS ( $2 \times 2$ repeated-measures ANOVA group-time interaction, $F_{(1,21)}=23.33, p=0.0001$; CUS at 3 weeks, ${ }^{*} p<0.05$ vs all other groups, Bonferroni test). $c$, AMPA/NMDAR ratios for TA-CA1 and SC-CA1 responses in each slice studied are plotted against the sucrose preference of the rat from which the slices were taken. TA-CA1 responses were correlated with sucrose preference $\left(R^{2}=0.36, p=\right.$ $0.025)$, whereas SC-CA1 responses were not $\left(R^{2}=0.003, p=0.43\right)$.

CUS exhibited a significantly lower sucrose preference than naive rats (Fig. 3b), and this behavioral measure had a significant positive correlation with TA-CA1 AMPA/NMDA ratios $(p=0.025$; Fig. 3c). Conversely, there was no significant correlation between SPT performance and AMPA/NMDA ratios of SC-CA1 synapses $(p=0.43$; Fig. $3 c)$.

We conclude that chronic stress-induced changes in behavior are strongly correlated with decreases in AMPAR-mediated synaptic excitation at TA-CA1 synapses, but not SC-CA1 synapses, as the result of changes in AMPAR expression and that restoring normal behavior with chronic selective serotonin reuptake inhibitor (SSRI) administration is accompanied by a recovery of AMPAR function and expression.

\section{CUS leads to a TA-CA1 synapse-specific cognitive deficit}

Does the impairment of excitatory synaptic transmission at TACA1 synapses have behavioral consequences? Previous work has shown that TA-CA1 synapses are required for long-term consolidation of spatial memory (Remondes and Schuman, 2004). We therefore asked whether subjecting animals to CUS after training them in a spatially cued version of the Morris water maze task would have any effect on memory consolidation. Naive rats were trained to learn the location of the platform successfully, and exhibited a preference for the target quadrant in a probe test $24 \mathrm{~h}$ after the last training trial (Fig. 4a). Rats were then randomly divided into CUS and control groups. Rats subjected to 3 weeks of CUS displayed a lower sucrose preference than did the unstressed controls, consistent with the induction of a depressive-like affective state. Twenty-eight days after training, the unstressed control rats performed as well in a probe trial as they did $24 \mathrm{~h}$ after the end of training, indicating that they had consolidated the memory of the platform location. Rats subjected to CUS, in contrast, showed a significantly lower preference for the target quadrant compared with $24 \mathrm{~h}$ post-training and compared with unstressed control rats (Fig. 4a), indicating that they failed to consolidate the memory of the platform location. This failure of consolidation thus mimicked the effect of TA-CA1 synapse ablation (Remondes and Schuman, 2004). There was no difference in swim speed, indicating that the CUS procedures did not produce motor deficits that affected their performance of the task (Fig. 4b). The performance of individual animals in the consolidation trial in the water maze was correlated positively with their individual SPT results $\left(r_{(14)}=\right.$ $0.50, p=0.07)$ and correlated negatively with the novelty- suppressed feeding test at the end of the experiment $\left(r_{(14)}=\right.$ $-0.74, p=0.002$; Fig. $4 c$ ).

\section{Discussion}

Stress is a significant risk factor for major depression, and is known to alter the dendritic architecture of neurons, including hippocampal pyramidal cells (Magariños and McEwen, 1995) but the functional consequences of these anatomical changes remain poorly understood. We report here that 3 weeks of CUS led to a decrease in the strength of excitatory synaptic transmission at TA synapses in the distal apical dendrites of CA1 cells. The decrease was specific to TA-CA1 synapses and was not detected at $\mathrm{SC}$ synapses in proximal dendrites.

The effects of chronic stress were limited to downregulation of AMPA-type glutamate receptors, without affecting NMDARs at the same synapses. The AMPAR-mediated component of the fEPSP was decreased by $\sim 50 \%$. The functional changes in synaptic transmission could be fully accounted for by the $\sim 50 \%$ decrease in GluA1 expression in SLM, but not SR. Consistent with our electrophysiological results, we observed no detectable changes in GluA2 or GluN1 expression after CUS. Our results contrast with those of Kole et al. (2002), who reported that 3 weeks of chronic restraint stress increased NMDAR-mediated responses in CA3 cells.

Long-term depression decreases the AMPAR-mediated component of the SC-CA1 EPSP, yet this decrease does not change the subsequent maximal synaptic strength that is reached after induction of LTP (Dudek and Bear, 1993). In contrast, the stressinduced decrease in AMPAR-mediated excitation was not accompanied by an increase in the relative magnitude of LTP at TA-CA1 synapses, indicating that the absolute value of postpotentiated synaptic strength is lower in CUS animals, along with the lower initial synaptic strength. Neither the biochemical machinery involved in the initiation and maintenance of LTP nor the relative size of the pool of AMPARs that can be rapidly recruited for membrane insertion appears perturbed following chronic stress. We suggest that activity-dependent synaptic plasticity remains capable of adjusting synaptic strength up or down at synapses after chronic stress. However, the range of potential synaptic strengths is scaled downward, thereby impairing the ability of TA inputs to trigger action potential output from area CA1.

Yuen et al. (2012) reported stress-induced decreases in synaptic excitation in pyramidal cells in the medial prefrontal cortex 
a
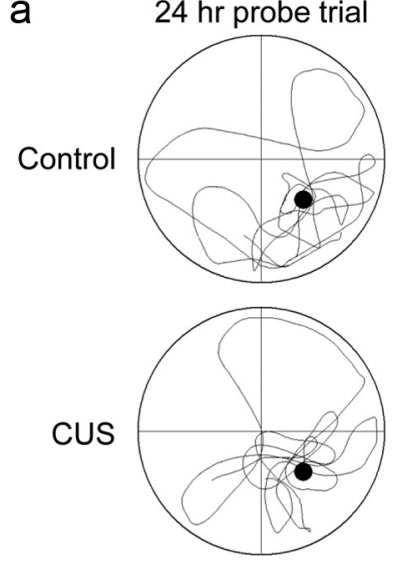

Consolidation trial
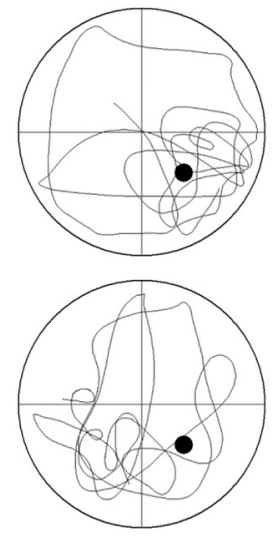
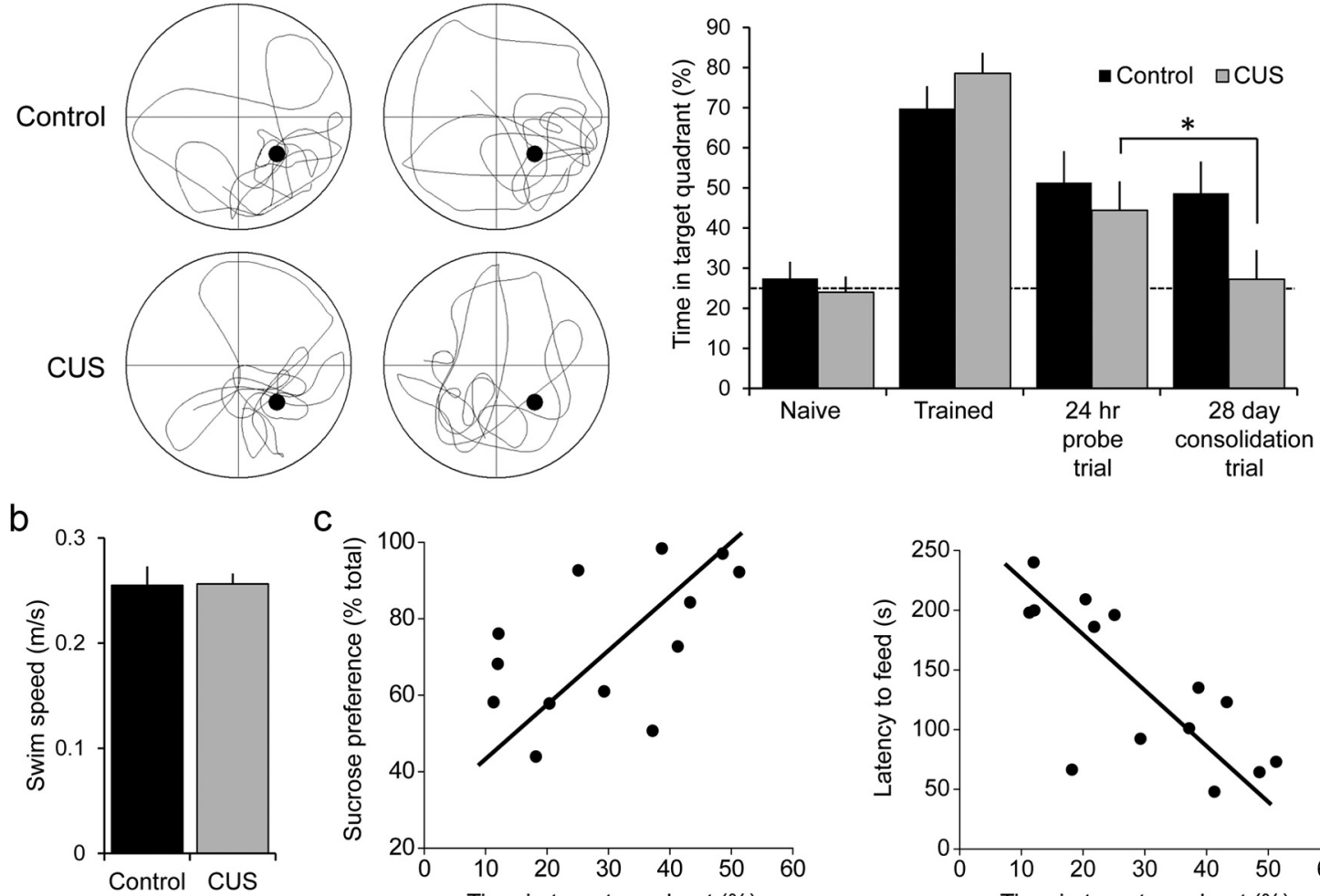

$\mathrm{C}$
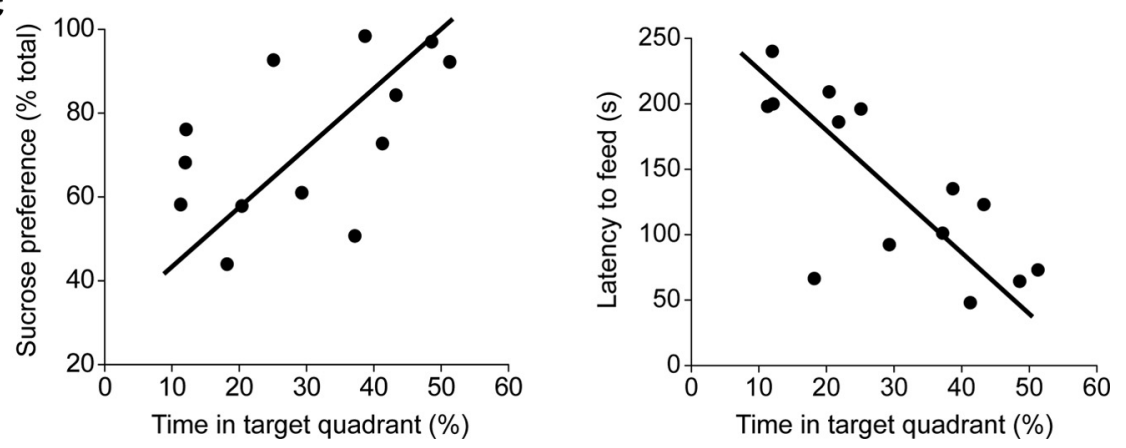

Figure 4. Chronic stress impairs consolidation of long-term memory. $\boldsymbol{a}$, Comparison of the percentage of time spent in the target quadrant during an initial probe trial, after training, in a memory probe trial performed $24 \mathrm{~h}$ after the last training trial, and a consolidation probe trial performed $28 \mathrm{~d}$ after the memory trial in control (black, $n=7$ ) and (US ( $g r a y, ~ n=6$ ) rats. There was a significant effect of time $\left(F_{(3,33)}=23.96, p<0.001\right)$ and a significant interaction $\left(F_{(3,33)}=3.07, p=0.042\right)$. Post hoc paired $t$ tests revealed no difference between the memory and consolidation probe times in quadrant for control animals $\left(t_{(5)}=0.2, p=0.85\right)$, but a significant difference for CUS animals $\left(t_{(6)}=3.44,{ }^{*} p=0.014\right)$. $\boldsymbol{b}$, There was no difference in swim speeds during the consolidation trial between control and CUS rats. $c$, The time in target quadrant in the consolidation trial for all animals was correlated with their sucrose preference (left, $\left.r_{(14)}=0.5, p=0.072\right)$ and latency to feed in the novelty-suppressed feeding test (right, $\left.r_{(14)}=-0.74, p=0.002\right)$.

(mPFC) mediated by degradation of GluA1 subunits. There are differences between these findings and ours, however. First, Yuen et al. (2012) found that both AMPARs and NMDARs were equally diminished in mPFC. They also observed no change in GluA1 function or expression in the hippocampus with $7 \mathrm{~d}$ of chronic restraint stress, a time at which GluA1 and GluN1 were decreased in $\mathrm{mPFC}$, suggesting that TA-CA1 synapses may be more resistant to the effects of chronic stress. Nevertheless, the commonalities of our study and that of Yuen et al. (2012) reinforces our suggestion that excitatory synapse dysfunction is an important contributing factor in stress-related neuropsychiatric disorders.

The decrease in synaptic efficacy at TA-CA1 synapses after chronic stress had deleterious behavioral consequences. Using long-term consolidation of spatially cued memory in the Morris water maze, a task that requires intact TA-CA1 synaptic transmission (Remondes and Schuman, 2004), we found that 3 weeks of CUS fully prevented memory consolidation. We suggest that not merely intact transmission and functional LTP, but a normal range of synaptic strength is required for TA-CA1 synapses to carry out their role in consolidation. If these synapses are weakened or effectively eliminated by chronic stress, then they appear incapable of subserving this function. Impaired spatial memory has also been described in depressed humans (Gould et al., 2007).

Results of individual animals in the consolidation trial were well correlated with the behavior of the animals in SPT and novelty-suppressed feeding tests, further highlighting the complex functional consequences of stress-induced synaptic weaken- ing. There was also a correlation between the magnitude of the decrease in the AMPA/NMDA ratio at TA-CA1 synapses in individual hippocampal slices with the behavior of the animals in the SPT. Although animals maintain a high sucrose preference in this test even after bilateral hippocampal lesions, the correlation we observed in these two disparate behaviors suggests that other populations of synapses respond to chronic stress similarly to TA-CA1 synapses, such as synapses in the mPFC (Yuen et al., 2012) and D1-expressing medium spiny neurons in the nucleus accumbens (Lim et al., 2012).

We have previously discovered that antidepressants exert an immediate potentiating action on excitatory synaptic transmission that is selective for TA-CA1, but not SC-CA1, synapses (Cai et al., 2013). This action is thus directly opposite to the effects of chronic stress. We now report that chronic $\mathrm{AD}$ administration in stressed animals also exerts a normalizing action on GluA1 expression and AMPAR-mediated excitation, suggesting that ADs exert long-term control of excitatory synaptic transmission.

The potentiating action of ADs at excitatory synapses was shown in our previous study to be necessary for the ability of these ADs to restore normal behaviors after chronic stress (Cai et al., 2013). The present results support our suggestion that normalization of synaptic excitation is a critical requirement of the therapeutic action of ADs. In this regard, it is noteworthy that ketamine exerts a rapid and lasting therapeutic response in depressed patients and a rapid strengthening of excitatory synaptic transmission through changes in AMPARs and expression of a variety of synaptic gene products (Berman et al., 2000; Maeng et 
al., 2008). Our observations offer a potential explanation for why ADs must be administered to depressed patients for weeks to months before their symptoms are alleviated. Perhaps the increased expression of GluA1 subunits is required in order for the acute potentiating actions of ADs to be able to exert lasting improvements in cognition and mood. Indeed, others have shown that chronic AD treatment increases AMPAR expression with a time course similar to therapeutic efficacy (Martinez-Turrillas et al., 2002).

Our data support an emerging excitatory synapse hypothesis of depression in which chronic stress decreases AMPAR function in specific subsets of synapses throughout the brain, of which TA-CA1 synapses represent a useful archetype. Depending on the anatomical location of the affected synapses, such glutamatergic dysfunction may account for the diverse symptoms of depression. Although serotonergic agents, such as SSRIs, are capable of producing therapeutic actions in depressed patients, we suggest that this fact does not necessarily indicate that a primary deficiency in serotonin synthesis or release is the cause of depression. Rather, AD-induced elevations in serotonin may exert beneficial actions indirectly as a result of increases in the strength of weakened excitatory synapses. Indeed, we found that chronic SSRI treatment rescued AMPAR dysfunction in chronically stressed animals. This suggestion is further supported by work showing that SSRIs can increase AMPAR phosphorylation and surface expression (Martinez-Turrillas et al., 2002; Svenningsson et al., 2002). In sum, these data provide a rationale for the exploration of new nonmonoaminergic therapeutic agents, including more compounds that directly and indirectly target the strength of excitatory synapses, such as AMPAkines and compounds that promote network excitability.

\section{References}

Alfarez DN, Joëls M, Krugers HJ (2003) Chronic unpredictable stress impairs long-term potentiation in rat hippocampal CA1 area and dentate gyrus in vitro. Eur J Neurosci 17:1928-1934. CrossRef Medline

Berman RM, Cappiello A, Anand A, Oren DA, Heninger GR, Charney DS, Krystal JH (2000) Antidepressant effects of ketamine in depressed patients. Biol Psychiatry 47:351-354. CrossRef Medline

Berton O, Nestler EJ (2006) New approaches to antidepressant drug discovery: beyond monoamines. Nat Rev Neurosci 7:137-151. CrossRef Medline

Bondi CO, Rodriguez G, Gould GG, Frazer A, Morilak DA (2008) Chronic unpredictable stress induces a cognitive deficit and anxiety-like behavior in rats that is prevented by chronic antidepressant drug treatment. Neuropsychopharmacol 33:320-331. CrossRef Medline

Cai X, Kallarackal AJ, Kvarta MD, Goluskin S, Gaylor K, Bailey AM, Lee HK, Huganir RL, Thompson SM (2013) Local potentiation of excitatory synapses by serotonin and its alteration in rodent models of depression. Nat Neurosci 16:464-472. CrossRef Medline

Dudek SM, Bear MF (1993) Bidirectional modification of synaptic effectiveness in the adult and immature hippocampus. J Neurosci 13:29102918. Medline

Gould NF, Holmes MK, Fantie BD, Luckenbaugh DA, Pine DS, Gould TD,
Burgess N, Manji HK, Zarate CA Jr (2007) Performance on a virtual reality spatial memory navigation task in depressed patients. Am J Psychiatry 164:516-519. CrossRef Medline

Kole MH, Swan L, Fuchs E (2002) The antidepressant tianeptine persistently modulates glutamate receptor currents of the hippocampal CA3 commissural associational synapse in chronically stressed rats. Eur J Neurosci 16:807-816. CrossRef Medline

Lim BK, Huang KW, Grueter BA, Rothwell PE, Malenka RC (2012) Anhedonia requires MC4R-mediated synaptic adaptations in nucleus accumbens. Nature 487:183-199. CrossRef Medline

Maeng S, Zarate CA Jr, Du J, Schloesser RJ, McCammon J, Chen G, Manji HK (2008) Cellular mechanisms underlying the antidepressant effects of ketamine: role of alpha-amino-3-hydroxy-5-methylisoxazole-4-propionic acid receptors. Biol Psychiatry 63:349-352. CrossRef Medline

Magariños AM, McEwen BS (1995) Stress-induced atrophy of apical dendrites of hippocampal CA3c neurons: comparison of stressors. Neuroscience 69:83-88. CrossRef Medline

Martinez-Turrillas R, Frechilla D, Del Río J (2002) Chronic antidepressant treatment increases the membrane expression of AMPA receptors in rat hippocampus. Neuropharmacology 43:1230-1237. CrossRef Medline

McEwen BS (1999) Stress and hippocampal plasticity. Annu Rev Neurosci 22:105-122. CrossRef Medline

Muscat R, Papp M, Willner P (1992) Reversal of stress-induced anhedonia by the atypical antidepressants, fluoxetine and maprotiline. Psychopharmacology 109:433-438. CrossRef Medline

Remondes M, Schuman EM (2004) Role for a cortical input to hippocampal area $\mathrm{CA} 1$ in the consolidation of a long-term memory. Nature 431:699-703. CrossRef Medline

Santarelli L, Saxe M, Gross C, Surget A, Battaglia F, Dulawa S, Weisstaub N, Lee J, Duman R, Arancio O, Belzung C, Hen R (2003) Requirement of hippocampal neurogenesis for the behavioral effects of antidepressants. Science 301:805-809. CrossRef Medline

Schmidt MV, Trümbach D, Weber P, Wagner K, Scharf SH, Liebl C, Datson N, Namendorf C, Gerlach T, Kühne C, Uhr M, Deussing JM, Wurst W, Binder EB, Holsboer F, Müller MB (2010) Individual stress vulnerability is predicted by short-term memory and AMPA receptor subunit ratio in the hippocampus. J Neurosci 30:16949-16958. CrossRef Medline

Sousa N, Lukoyanov NV, Madeira MD, Almeida OF, Paula-Barbosa MM (2000) Reorganization of the morphology of hippocampal neurites and synapses after stress-induced damage correlates with behavioral improvement. Neuroscience 97:253-266. CrossRef Medline

Svenningsson P, Tzavara ET, Witkin JM, Fienberg AA, Nomikos GG, Greengard P (2002) Involvement of striatal and extrastriatal DARPP-32 in biochemical and behavioral effects of fluoxetine (Prozac). Proc Natl Acad Sci U S A 99:3182-3187. CrossRef Medline

Vyas A, Mitra R, Shankaranarayana Rao BS, Chattarji S (2002) Chronic stress induces contrasting patterns of dendritic remodeling in hippocampal and amygdaloid neurons. J Neurosci 22:6810-6818. Medline

Watanabe Y, Gould E, McEwen BS (1992) Stress induces atrophy of apical dendrites of hippocampal CA3 pyramidal neurons. Brain Res 588: 341-345. CrossRef Medline

Willner P, Towell A, Sampson D, Sophokleous S, Muscat R (1987) Reduction of sucrose preference by chronic unpredictable mild stress, and its restoration by a tricyclic antidepressant. Psychopharmacology 93:358-364. Medline

Yuen EY, Wei J, Liu W, Zhong P, Li X, Yan Z (2012) Repeated stress causes cognitive impairment by suppressing glutamate receptor expression and function in prefrontal cortex. Neuron 73:962-977. CrossRef Medline 